\title{
Coordination of tRNA nuclear export with processing of tRNA
}

\author{
GERD LIPOWSKY, ${ }^{1}$ F. RALF BISCHOFF, ${ }^{2}$ ELISA IZAURRALDE, ${ }^{3}$ ULRIKE KUTAY, ${ }^{1}$ \\ STEFAN SCHÄFER, ${ }^{4}$ HANS J. GROSS, ${ }^{4}$ HILDBURG BEIER, ${ }^{4}$ and DIRK GÖRLICH ${ }^{1}$ \\ ${ }^{1}$ Zentrum für Molekulare Biologie der Universität Heidelberg, INF 282, 69120 Heidelberg, Germany \\ ${ }^{2}$ Abteilung Molekulare Biologie der Mitose, Deutsches Krebsforschungszentrum, INF 280, 69120 Heidelberg, Germany \\ ${ }^{3}$ University of Geneva, Department of Molecular Biology, CH-1211 Geneva 4, Switzerland \\ ${ }^{4}$ Institut für Biochemie, Biozentrum der Universität Würzburg, Am Hubland, 97074 Würzburg, Germany
}

\begin{abstract}
Eukaryotic tRNAs are synthesized in the nucleus and need to be exported to the cytoplasm where they function in translation. tRNA export is mediated by exportin-t, which binds tRNA directly and with high affinity. tRNAs are initially synthesized as precursor molecules. Maturation to functional tRNA takes place in the nucleus, precedes export, and includes trimming of the $5^{\prime}$ and $3^{\prime}$ ends, posttranscriptional addition of the $3^{\prime}$ CCA end, nucleoside modifications, and in some cases splicing. Here we address the question of how tRNA maturation is coordinated with export and thus how cytoplasmic accumulation of inactive maturation intermediates is avoided. This could, in principle, be achieved by nuclear retention of immature tRNA or by selective export of the fully mature form. We show that exportin-t has a strong preference for tRNA with correctly processed $5^{\prime}$ and $3^{\prime}$ ends and nucleoside modification. tRNA recognition by exportin-t can thus be considered as a quality control mechanism for these maturation steps prior to tRNA export. Surprisingly however, exportin-t can efficiently bind unspliced tRNA and intron-containing tRNA is exported when the rate of splicing is slow. During characterization of the exportin-t/tRNA interaction we found that exportin-t recognizes features in the tRNA that are conserved between prokaryotic and eukaryotic tRNAs. Our data suggest that correct tRNA shape, the $5^{\prime}$ and $3^{\prime}$ terminal ends, and the T $\Psi C$ loop are critical for exportin-t binding.
\end{abstract}

Keywords: exportin-t; nuclear pore complex; nuclear transport; tRNA; tRNA maturation

\section{INTRODUCTION}

The division of eukaryotic cells into a nuclear and cytoplasmic compartment by the nuclear envelope (NE) necessitates constitutive and regulated transport between the two compartments. For example, all nuclear proteins are made in the cytoplasm and need to be imported. Conversely, transcription is nuclear and tRNA, mRNA, and rRNA need to be exported to the cytoplasm where they function in translation. All nucleocytoplasmic transport proceeds through nuclear pore complexes (NPCs) that allow diffusion of small molecules and accommodate active transport of particles up to several million daltons in molecular weight. This active transport is largely mediated by shuttling transport receptors (for recent reviews see Dahlberg \& Lund, 1998; Izaurralde \& Adam, 1998; Mattaj \& Englmeier, 1998). A number of import receptors (importins) and three exportins, namely CAS,

Reprint requests to: D. Görlich, Zentrum für Molekulare Biologie der Universität Heidelberg, INF 282, 69120 Heidelberg, Germany; e-mail: dg@mail.zmbh.uni-heidelberg.de.
Crm1p, and exportin-t, have been identified so far. All transport receptors are, at least distantly, related to importin $\beta$ and function according to common principles. They bind cargo on one side of the nuclear envelope, mediate translocation through NPCs, release the cargo on the other side, and then return to the original compartment.

The loading and unloading of cargo is coordinated by the RanGTPase system. Ran itself can switch between a GTP-bound state and a GDP form by GTP hydrolysis and nucleotide exchange. These transitions are catalyzed by the GTPase activating protein RanGAP1 that is excluded from the nucleus and thus depletes RanGTP from the cytoplasm and by the chromatin-bound nucleotide exchange factor RCC1 that generates RanGTP in the nucleus. This is thought to result in a RanGTP gradient across the nuclear envelope with a high RanGTP concentration in the nucleus and low levels in the cytoplasm. Transport receptors are RanGTP-binding proteins and are therefore capable of responding to this gradient by loading and unloading their cargo in the appropriate compartment. 
An exportin has a high affinity for its substrate when bound to RanGTP (Fornerod et al., 1997; Kutay et al., 1997; Arts et al., 1998a; Kutay et al., 1998). Its substrate affinity is low when in the Ran-free state. Exportin-t, for example, binds tRNA together with RanGTP in the nucleus to form a trimeric complex that is subsequently exported and finally disassembled in the cytoplasm. This disassembly is brought about by RanGAP1 and RanBP1 and involves release of the tRNA and the hydrolysis of the Ran-bound GTP. The cargo-free exportin can then re-enter the nucleus and bind and export the next tRNA molecule (Arts et al., 1998a; Kutay et al., 1998).

The existence of a tRNA export receptor was predicted 15 years ago from microinjection experiments (Zasloff, 1983). Zasloff found that tRNA export from nuclei of Xenopus oocytes is a saturable process and, by this criterion, carrier-mediated. It was later shown that the tRNA export pathway is specific for this class of export substrates as it could be competed by an excess of the tRNA itself, but not by mRNAs or $U$ snRNAs (Jarmolowski et al., 1994). It is important to note that exportin-t binds tRNA directly and indeed accounts for the class-specificity of export predicted by these earlier experiments (Arts et al., 1998a; Kutay et al., 1998). It should also be noted that tRNA export is conserved between lower and higher eukaryotes, the yeast homolog of exportin-t being Los1p (Hopper et al., 1980; Hurt et al., 1987; Simos et al., 1996; Arts et al., 1998a; Hellmuth et al., 1998; Kutay et al., 1998; Sarkar \& Hopper, 1998).

tRNAs are initially synthesized as precursors (pre-tRNAs). Their maturation to functional tRNA includes trimming of the $5^{\prime}$ and $3^{\prime}$ ends, modification of a number of nucleosides, the posttranscriptional addition of the 3' CCA end, and in some cases also the removal of a small intron (for recent reviews, see Björk, 1995; Deutscher, 1995; Sprague, 1995; Westaway \& Abelson, 1995). Microinjection experiments have shown that most of these processing events take place in the nucleus (Melton et al., 1980; De Robertis et al., 1981). tRNA maturation obviously needs to be properly coordinated with nuclear export in order to avoid cytoplasmic accumulation of immature and nonfunctional tRNA.

To determine how tRNA maturation and export may be coordinated, we have investigated the interaction of exportin-t with a number of (putative) maturation intermediates of tRNA ${ }^{\text {Lys } 3}$. We found that exportin-t has a clear preference for the fully mature tRNA. In particular, correctly processed $5^{\prime}$ and $3^{\prime}$ ends, including the $3^{\prime}$ CCA addition, are critical for high-affinity interaction with exportin-t. Furthermore, modified nucleosides apparently play a role in binding to exportin-t. tRNA splicing precedes export in vivo. Surprisingly, however, an intron in tRNA ${ }^{\text {Tyr }}$ does not interfere with exportin-t binding. Moreover, nuclear export of intron-containing tRNA can occur efficiently, provided splicing is slow. Thus the temporal coordination of splicing with export might be controlled kinetically. Analysis of tRNA point mutants revealed that the TUC loop and tertiary tRNA structure are critical for interaction with the export receptor. Mutations in the tRNA that interfere with exportin-t binding also caused slow export. Finally, we show that exportin-t recognizes features conserved between eukaryotic and prokaryotic tRNAs, suggesting that exportin-t has adapted in evolution to preexisting tRNA-features and not the other way round.

\section{RESULTS}

Exportin-t has recently been identified as a specific mediator of tRNA export from the cell nucleus (Arts et al., 1998a; Kutay et al., 1998). We have previously shown that exogenous exportin-t stimulates tRNA export from nuclei of Xenopus oocytes. Although this experiment clearly showed the potential of exportin-t to export tRNA, it could not formally exclude the possibility that exportin-t only represents a minor tRNA export pathway in vivo. To clarify the issue, we injected monospecific antibodies raised against exportin-t or a control antibody into nuclei of Xenopus oocytes and studied the effect on export of several coinjected RNAs. If buffer or the control antibody were preinjected, then $\operatorname{tRNA} A_{i}^{\text {Met }}$ was exported to reach a cytoplasmic:nuclear ratio of 4.5:1 (Fig. 1). After preinjection of the anti-exportin-t antibody, this ratio dropped to $1: 1$, but export of $U 1$ snRNA and DHFR mRNA remained unaffected. The anti-exportin-t antibodies also specifically inhibited export of other tRNA species such as tRNA ${ }^{\text {Ser }}$ (data not shown). Thus, one can conclude that the majority of tRNA is indeed exported by exportin-t.

\section{Exportin-t recognizes tRNA features that are conserved between prokaryotes and eukaryotes}

The actual tRNA export complex is trimeric and assembles in a highly cooperative manner from exportin-t, the tRNA, and RanGTP. We were interested in the distinctive features of a tRNA that make it a substrate for exportin-t. tRNA is evolutionary more ancient than are nuclei and thus the nuclear transport machinery. An obvious question is whether the nuclear export machinery has adapted to these ancient, conserved tRNA characteristics. Alternatively, eukaryotic tRNA could have acquired novel features that are essential for export and not yet present in prokaryotic tRNA. To distinguish between the two possibilities, we compared the capacity of eukaryotic and prokaryotic tRNA to engage into trimeric tRNA/exportin-t/RanGTP complexes.

As seen from Figure 2A, tRNA from calf liver, Saccharomyces cerevisiae, or Escherichia coli all bound to exportin-t in a RanGTP-dependent manner. The specificity of binding was verified by two controls. First, ef- 


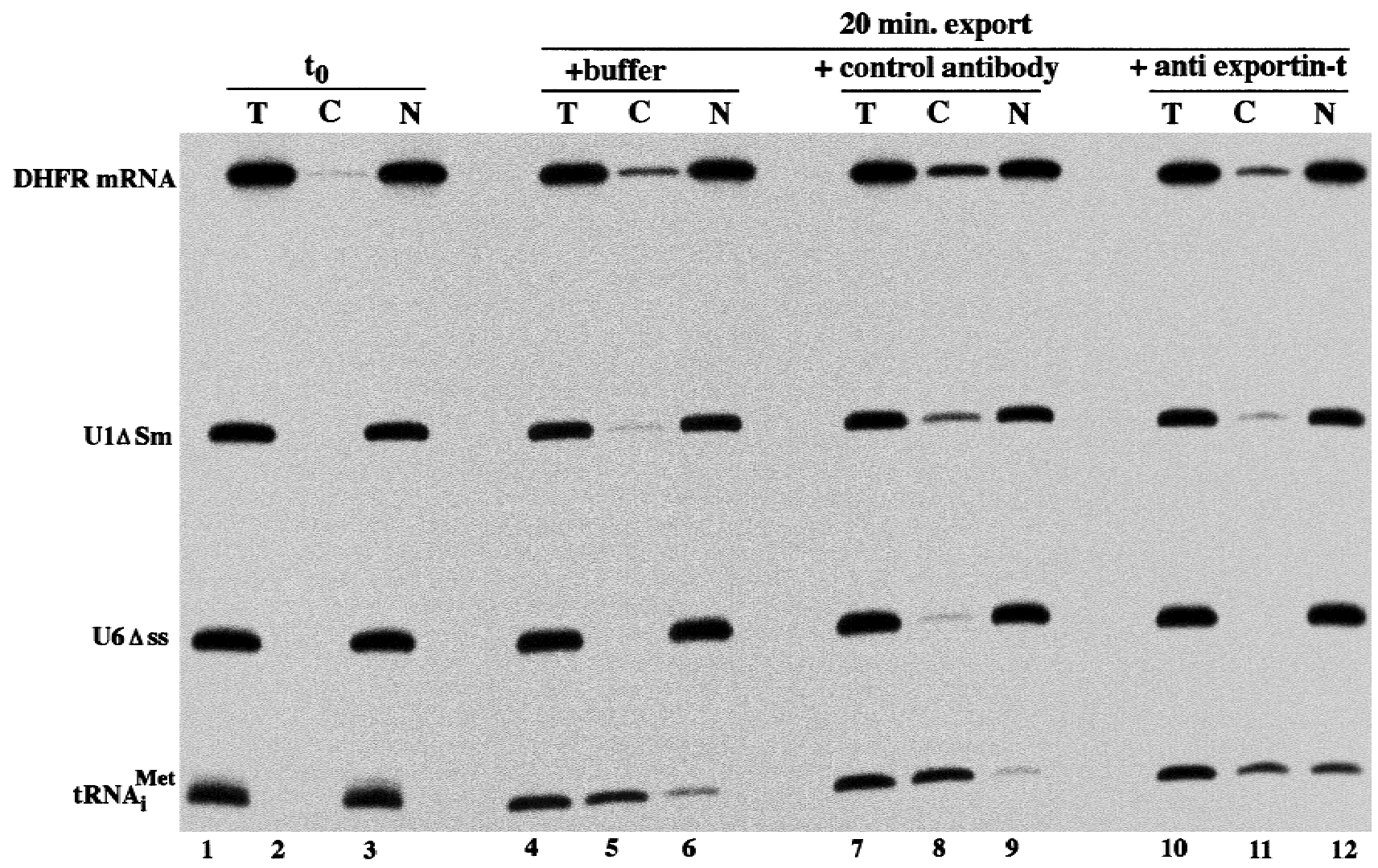

FIGURE 1. Inhibition of tRNA export by anti-exportin-t antibodies. The nuclei of Xenopus laevis oocytes were preinjected with either buffer (lanes 1-6), $0.2 \mu \mathrm{g}$ control antibody (lanes 7-9), or $0.2 \mu \mathrm{g}$ of a monospecific antibody raised against exportin-t (lanes 10-12). Two hours later, the nuclei were injected with the indicated mixture of ${ }^{32} \mathrm{P}$-labeled RNAs. RNA samples from total oocytes $(\mathrm{T})$ or cytoplasmic $(\mathrm{C})$ and nuclear $(\mathrm{N})$ fractions were collected either immediately after injection of the RNA mixture $\left(t_{0}\right.$, lanes 1-3) or after 20 min further incubation (lanes 4-12). RNAs were resolved on an 8\% polyacrylamide-7 M urea denaturing gel and detected by autoradiography. Note that the control antibody (lanes 7-9) had no effect on tRNA export (ratio of cytoplasmic tRNA to nuclear tRNA $=4.5$ ). In contrast, the injection of the anti-exportin-t antibody (lanes 10-12) selectively inhibited export of tRNA $A_{i}^{\text {Met }}$ (cytoplasmic tRNA:nuclear tRNA $=1$ ). U6 $s$ ss is neither imported nor exported and served as an internal control for proper injection and nuclear integrity.

ficient binding to immobilized exportin-t was only observed in the presence of RanGTP (here supplied as RanQ69L GTP), whereas RanGDP (T24N-GDP) had no effect. Second, U6 $\Delta$ ssRNA was not detectably bound under these conditions.

To obtain more quantitative data, we employed a kinetic assay to measure the formation of the trimeric complex. Binding of a $\beta$-like transport receptor to RanGTP prevents GTPase activation by RanGAP1 (Floer \& Blobel, 1996; Görlich et al., 1996). GTP hydrolysis can easily be quantified and used to calculate the proportion of Ran bound to, for example, exportin-t. Binding of RanGTP and tRNA to exportin-t is highly cooperative in that tRNA increases the affinity of exportin-t for RanGTP roughly 300 -fold and vice versa (Kutay et al., 1998). From the dose dependence of the effects, one can estimate apparent dissociation constants $\left(K_{d} \mathrm{~s}\right)$ for the complex formation. As seen from Figure 2B, tRNA from calf liver, yeast, and $E$. coli bound to exportin-t equally well and gave superimposable binding curves with a half-maximum effect at about $3 \mathrm{nM}$.
Consistent with these results we found that $E$. colitRNA can efficiently compete tRNA export from Xenopus oocyte nuclei (not shown). Taken together, we conclude that exportin-t recognizes features of tRNA that are highly conserved between eukaryotes and prokaryotes.

\section{Exportin-t preferentially binds mature tRNA}

tRNAs are initially transcribed as larger precursors with $5^{\prime}$ leader and $3^{\prime}$ trailer sequences and in some cases (such as tRNA ${ }^{\text {Tyr }}$ ) also with an intron. Maturation of tRNA involves removal of the $5^{\prime}$ and $3^{\prime}$ extensions, splicing, modification of a number of nucleosides, and the posttranscriptional generation of the $3^{\prime}$ CCA end to which the appropriate amino acids will later be attached (for recent reviews, see Björk, 1995; Deutscher, 1995; Sprague, 1995; Westaway \& Abelson, 1995). Microinjections of tRNA ${ }^{T y r}$ precursors into nuclei of Xenopus oocytes have clearly shown that most enzymatic activities required for tRNA maturation are nuclear and that indeed most maturation steps are 

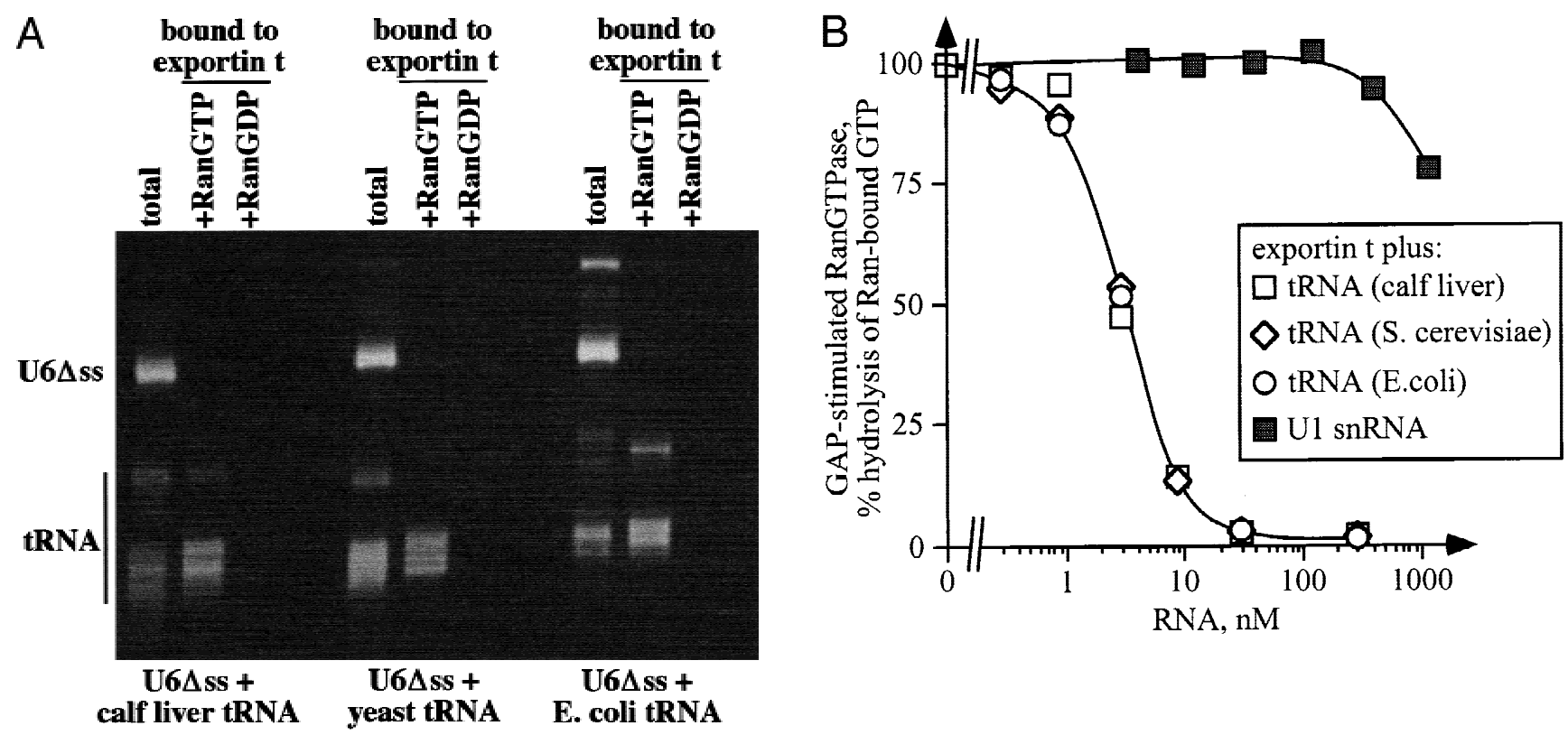

FIGURE 2. Exportin-t binds tRNA from eukaryotes and prokaryotes. A: $500 \mathrm{pmol}$ total tRNA from calf liver, $S$. cerevisiae, or $E$. coli were each mixed with $500 \mathrm{pmol} U 6 \Delta \mathrm{ss}$ RNA and subjected to binding to immobilized exportin-t. Binding was either in the presence of $6 \mu \mathrm{M}$ RanQ69L GTP or RanT24N GDP. After extensive washing, bound fractions were recovered by phenol/chloroform extraction and analyzed by denaturing gel electrophoresis and SYBR-GOLD staining. Load was 1/20 of the starting material and $1 / 10$ of the bound fractions. Note that tRNA from all three species bound specifically to exportin-t in a RanGTP-dependent manner. B: Panel shows binding curves for assembly of tRNA into tRNA/exportin-t/RanGTP complexes. The assay is based on the observations that RanGTP present in such complex resists GTPase activation by RanGAP1. $50 \mathrm{pmol} \gamma^{32} \mathrm{P}$ RanGTP were incubated for $15 \mathrm{~min}$ with a mixture of $40 \mathrm{nM}$ exportin-t and the various nonacylated tRNAs (calf-liver, S. cerevisiae, E. coli) or U1snRNA (in vitro transcribed) at indicated final concentrations. GTPase activation was induced by addition of RanGAP1 and released ${ }^{32} \mathrm{P}$ phosphate was determined 2 min later as described (Bischoff et al., 1995; Kutay et al., 1998). The formation of the tRNA/exportin-t/RanGTP complex was measured as the decrease in GAP sensitivity of RanGTP. Note that tRNA from calf liver, $S$. cerevisiae, and $E$. coli bound to exportin-t equally well. The half-maximum effect (apparent $K_{d}$ ) was at approximately $3 \mathrm{nM}$ tRNA.

completed before the tRNA is exported to the cytoplasm (Melton et al., 1980; De Robertis et al., 1981). This coordination of tRNA maturation and export could, in principle, be achieved either by nuclear retention of the immature tRNA, by a preference of the export receptor for the mature tRNA, or by a combination of both.

We have previously presented data suggesting that nucleoside modifications in the tRNA might be crucial for high-affinity interaction between tRNA, exportin-t, and RanGTP. We found that fully modified, total cytoplasmic tRNA would bind to exportin-t more avidly than single in vitro-transcribed species that lacked those modifications (Kutay et al., 1998). Although this experiment strongly suggested that the nucleoside modifications are important, it did not rule out the formal alternative interpretation that different tRNA species have different affinities independent of such modifications and that by chance we had in vitro-transcribed those that bind more weakly. To clarify this point, we decided to compare exportin-t binding of various (putative) maturation intermediates of a single tRNA species, namely tRNA ${ }^{\text {Lys3 }}$ and used for this purpose the quantitative, kinetic assay described above. The fully modified form, purified from bovine liver, bound with the highest affinity. For easy comparison, Table 1 lists relative affinities. An in vitro-transcribed tRNA with mature $5^{\prime}$ and $3^{\prime}$ ends had only $18 \%$ of the affinity of the fully mature form. The only difference between the two is the presence of nucleoside modifications in the mature form, suggesting that these modified nucleosides indeed significantly stabilize the tRNA-exportin-t complex.

\section{Only tRNA with correctly processed $5^{\prime}$ and $3^{\prime}$ ends binds efficiently to exportin-t}

Table 1 also shows that the presence of a $5^{\prime}$ leader or a $3^{\prime}$ trailer on the tRNA severely interferes with exportin-t binding, suggesting that these extensions have to be removed before the tRNA can be exported. The partial or complete deletion of the $3^{\prime}$ CCA end had a similar negative effect on exportin-t binding (Table 2). Surprisingly, however, when the absent CCA end was replaced by UUU, exportin-t binding was partially restored. It thus appears that the correct length of the $3^{\prime}$ tRNA end is more crucial than its actual sequence for highaffinity interaction with exportin-t. 
TABLE 1.

\begin{tabular}{llcc}
\hline tRNA species (Lys 3) & & $\begin{array}{c}\text { Relative affinities } \\
\text { Contains modifications? }\end{array}$ & $\begin{array}{c}\text { for exportin-t/RanGTP } \\
\text { Native (from bovine liver) }\end{array}$ \\
In vitro transcribed & mature ends & yes & 100 \\
& mature ends & no & 18 \\
& with 10 nt 5' extension & no & 2.4 \\
& with 10 nt 3' extension & no & 7.2 \\
& with 16 nt 3' extension & no & no \\
& with 10 nt 3' and 10 nt 5' extensions & no & 0.8 \\
& without 3' CCA end & 2.4 \\
\hline
\end{tabular}

Apparent $K_{d} \mathrm{~S}$ for interaction of the indicated tRNA ${ }^{\text {Lys } 3}$ species with exportin-t were determined as described in Figure $2 \mathrm{~B}$. Affinities are inversely proportional to the $K_{d} \mathrm{~s}$. Affinity of the fully mature form was set to 100 .

\section{Exportin-t does not discriminate between intron-containing, already spliced, or intronless tRNA}

Some tRNA precursors such as pre-tRNA ${ }^{T y r}$, pre-tRNA $A^{\text {Trp }}$, or pre-tRNA ${ }^{\text {Phe }}$ contain an intron. The length of the intron is variable (8-60 nt), but its position, $1 \mathrm{nt}$ downstream from the anticodon, is conserved in eukaryotes (Sprinzl et al., 1989; Westaway \& Abelson, 1995; Belfort \& Weiner, 1997; for review, see Abelson et al., 1998). tRNA splicing is a nuclear event that precedes export (Melton et al., 1980; De Robertis et al., 1981; see also Bertrand et al., 1998; Pederson, 1998, and references therein; Sarkar \& Hopper, 1998). It was therefore interesting to know if the presence of the intron would affect exportin-t binding. To test this, we in vitro-transcribed tRNA ${ }^{\text {Tyr }}$ with or without an intron (see Fig. 3 for a scheme) and measured the formation of the trimeric tRNA/exportin-t/RanGTP complexes by the kinetic assay described above. Surprisingly, both tRNAs bound with virtually identical affinity (Fig. 4). Thus, exportin-t cannot distinguish between intron-containing and intronless tRNA. In addition, the experiment suggests that the anticodon loop, into which the intron is inserted, probably does not contribute to exportin-t binding.

If exportin-t can efficiently bind intron-containing tRNA, then such tRNA should also be a nuclear export substrate. When intron-containing tRNA ${ }^{\text {Tyr }}$ with a $5^{\prime}$ leader (Zerfass \& Beier, 1992; Akama et al., 1997; see Fig. 3B) was injected into Xenopus oocyte nuclei, then trimming of the $5^{\prime}$ end and splicing were very efficient

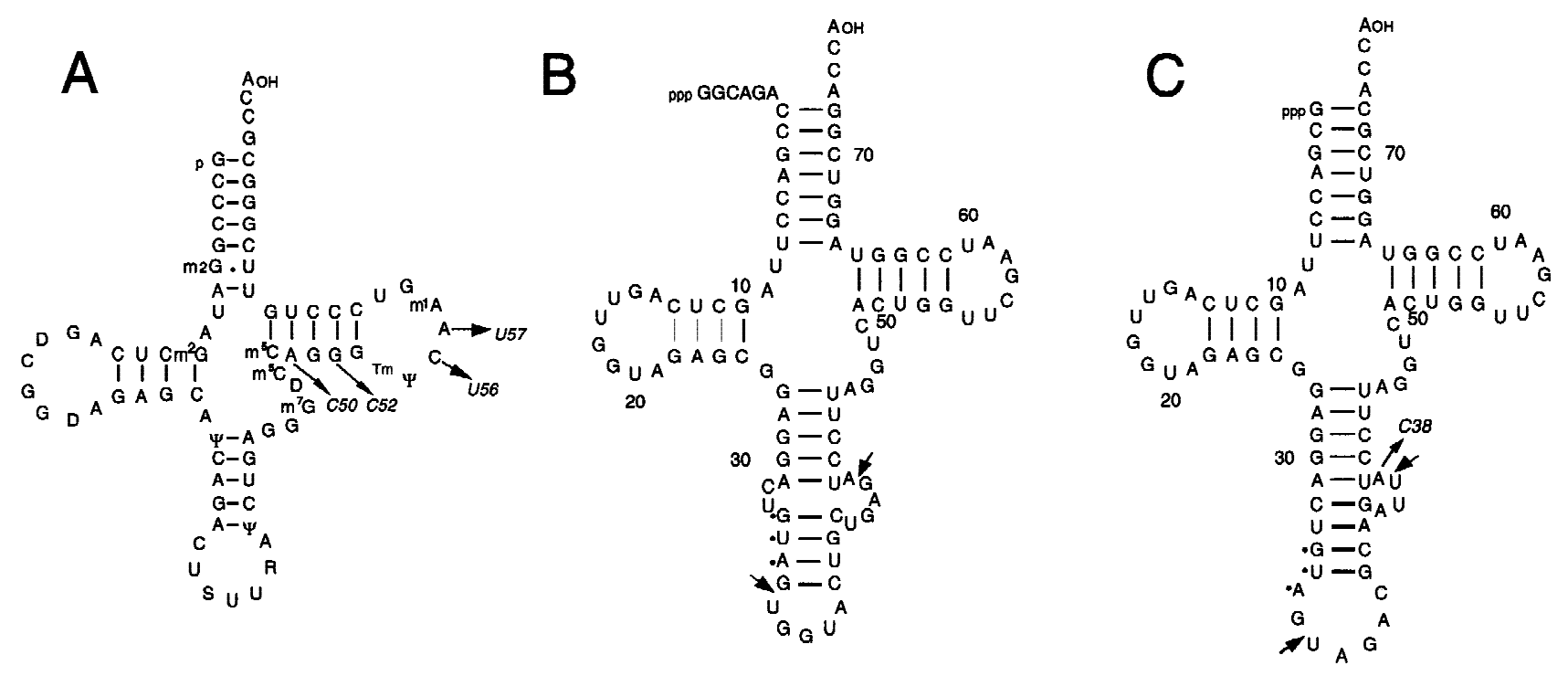

FIGURE 3. Secondary structures of mature tRNA $A^{\text {Lys3 }}$ and intron-containing pre-tRNA ${ }^{T y r}$ species. A: Nucleotide sequence of cytoplasmic tRNA ${ }^{\mathrm{Lys} 3}$ from bovine liver that is identical in sequence to that from rabbit liver (Raba et al., 1979). S: $\mathrm{mcm}^{5} \mathrm{~s}^{2} \mathrm{U}$. R: $\mathrm{ms}^{2} \mathrm{t}^{6} \mathrm{~A}$. The nucleotide substitutions introduced into tRNA ${ }^{\text {Lys3 }}$ are A50 to C, G52 to C, C56 to U, and A57 to U. B: Nicotiana pre-tRNA ${ }^{\mathrm{Tyr}}$ derived from pNtYl-T7 (Zerfass \& Beier, 1992) carries an intron of 13 nt. C: Arabidopsis pre-tRNA ${ }^{\mathrm{Tyr}}$ derived from pAtY3II*-T7 (Akama et al., 1997) carries an intron of 12 nt. The introduced mutation (i.e., A38 to C) results in a splicing-defective pre-tRNA. Arrows point to $5^{\prime}$ and $3^{\prime}$ splice sites; dots identify the anticodons. 
TABLE 2.

\begin{tabular}{lc}
\hline 3' CCA end of tRNA & $\begin{array}{c}\text { Relative affinities } \\
\text { for exportin-t/RanGTP }\end{array}$ \\
\hline CCA (no change) & 100 \\
CC & 57 \\
C & 28 \\
without CCA & 10 \\
UUU & 59 \\
\hline
\end{tabular}

Relative affinities of the indicated tRNA species were determined as described in Table 1 and Figures $2 B$ and 4 . tRNAs were generated by in vitro transcription, had mature $5^{\prime}$ ends, and differed only at the $3^{\prime}$ end.

and essentially complete by $60 \mathrm{~min}$, and most of the mature tRNA ${ }^{\text {Tyr }}$ had reached the cytoplasm (Fig. 5). When an intron-containing tRNA ${ }^{\text {Tyr }}$ was injected without the $5^{\prime}$ leader, then splicing was less efficient and remained incomplete. Strikingly, the unspliced, introncontaining tRNA ${ }^{\text {Tyr }}$ was now very efficiently exported. Furthermore, tRNA ${ }^{\text {Tyr }}$ with a point mutation that blocks splicing (C38; see Fig. 3C) was also exported (Fig. 5). In summary, exportin-t can efficiently bind and export intron-containing tRNA. The temporal coordination of tRNA splicing and export might thus be kinetically controlled (for discussion, see below).

\section{Conserved nucleotides in the T stem-loop regions of the tRNA are critical for exportin-t binding}

The cloverleaf secondary structure of tRNA folds further into a characteristic L-shaped tertiary structure, whereby tertiary interactions between highly conserved nucleotides play a critical role (for review, see Dirheimer et al., 1995). In particular, an interaction between the $D$ and the TIC loop is crucial to give the tRNA its correct shape. Mutations in such critical nucleotides severely affect tRNA processing but also export of an eventually matured tRNA (Tobian et al., 1985). Impaired export could be because of nuclear retention of the mutant tRNA as suggested by Boelens et al. (1995) or because of an inefficient interaction with exportin-t. To address this question, we tested several mutants of tRNA ${ }^{\text {Lys3 }}$ (see Fig. $3 A$ ) with correct $5^{\prime}$ and $3^{\prime}$ ends for exportin-t binding, using the quantitative kinetic assay described above (Table 3). The A50C and G52C mutations disturb the secondary structure in the $T$ stem and reduced exportin-t binding by a factor of 40 and 120 , respectively. Combining the two mutations resulted in a further reduction in affinity. C56 is involved in a tertiary base pairing between the T and the $\mathrm{D}$ loop. Changing this nucleotide to $\mathrm{U}$ abolishes this interaction and reduced the affinity for exportin-t roughly 40 -fold (table 3), emphasizing that a correct tRNA shape is critical for exportin-t binding.

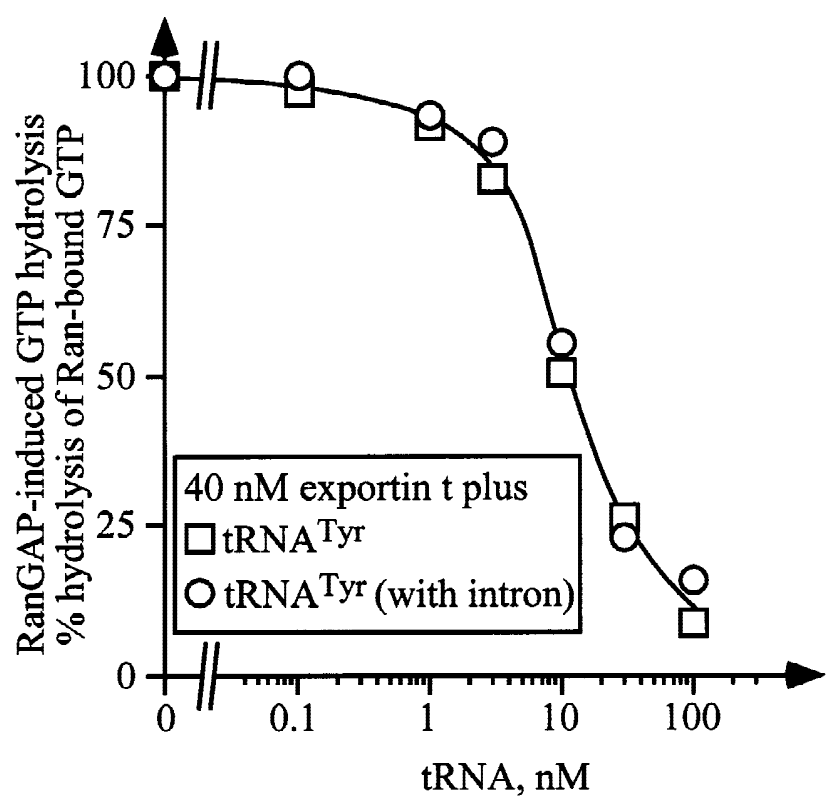

FIGURE 4. An intron does not interfere with the tRNA/exportin-t interaction. tRNA ${ }^{\mathrm{Tyr}}$ with mature $5^{\prime}$ and $3^{\prime}$ ends and either with or without an intron was obtained by in vitro transcription (Zerfass \& Beier, 1992). Binding to exportin-t was measured by the kinetic assay described in Figure $2 \mathrm{~B}$. Note that intron-containing and intronfree tRNA ${ }^{T y r}$ gave superimposable binding curves.

An interesting mutant is $\mathrm{A} 57 \mathrm{U}$, which was first described for tRNA $A_{i}^{\text {Met }}$ as blocking tRNA maturation and export (Zasloff et al., 1982a, 1982b). This base is located in the TYC loop, but apparently does not contribute to secondary or tertiary structure formation. We have examined the analogous mutation in tRNA ${ }^{\text {Lys3 }}$ and as seen from Table 3, this mutation also drastically interferes with exportin-t binding. The T $\Psi C$ loop therefore appears to be part of the tRNA signature that is "read" by exportin-t.

Finally, we tested nuclear export of two of the point mutants (A57U and A50C,G52C) following microinjection into nuclei of Xenopus oocytes. As seen from Figure 6 , the mutations essentially abolished export, as was expected from their poor binding to exportin-t.

\section{DISCUSSION}

Eukaryotic tRNAs are initially synthesized as pre-tRNAs in the nucleus, processed to mature tRNA, and exported to the cytoplasm. There, they participate in cycles of aminoacylation, binding to the elongation factor eE1A, and function in translation. Each of these events involves a very specific tRNA recognition. However, the constraints are very different for the interaction of tRNA with the various tRNA-binding factors. The elongation factor eE1A, for example, has to recognize all aminoacylated tRNAs needed for translational elongation, but discriminate against the initiator tRNA and nonaminoacylated tRNA. Aminoacyl tRNA synthetases have 


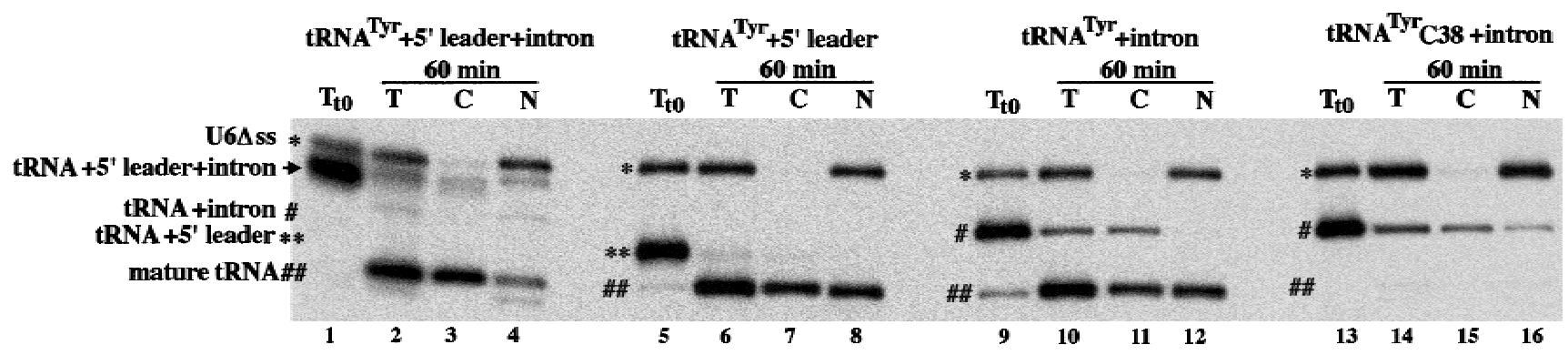

FIGURE 5. Intron-containing tRNA can be efficiently exported from nuclei of Xenopus oocytes. Nuclei of Xenopus oocytes were injected with ${ }^{32} \mathrm{P}$-labeled U6 $\Delta \mathrm{sS}$ RNA (as an injection control) and with the tRNA ${ }^{\mathrm{Tyr}}$ derivatives indicated above the lanes. RNAs were collected either immediately after injection $\left(t_{0}\right)$ or after 60 min incubation. T: total oocytes; C: cytoplasmic fraction; N: nuclear fraction. Note that $5^{\prime}$ processing and splicing were essentially complete for tRNA ${ }^{\text {Tyr }}$ containing a $5^{\prime}$ leader and an intron and export of tRNA was very efficient (lanes 1-4). Splicing of tRNA ${ }^{\mathrm{Tyr}}$ containing an intron but lacking the $5^{\prime}$ extension was incomplete and the nonspliced tRNA was very efficiently exported to the cytoplasm (lanes 9-10). C38 (lanes 13-16) is a mutation that blocks splicing (see Fig. 3C; Akama et al., 1997).

to be highly specific for a given isoacceptor. In contrast, the nuclear export machinery should recognize virtually all tRNA species.

tRNAs are small enough to diffuse through nuclear pore complexes, but already in 1983 it was demonstrated that nuclear export of tRNA is normally mediated by a saturable carrier (Zasloff, 1983). Exportin-t has recently been identified as such a tRNA export mediator (Arts et al., 1998a; Kutay et al., 1998). Exportin-t binds tRNA specifically, tRNA export from nuclei of Xenopus oocyte is stimulated by exogenous exportin-t (Arts et al., 1998a; Kutay et al., 1998) and is specifically inhibited by antibodies raised against exportin-t (Fig. 1; see also Arts et al., 1998b). Furthermore, there is a good correlation between the affinity of a given tRNA species for exportin-t and its actual export. For example, the A57U mutation in tRNA ${ }^{\text {Lys3 }}$ interferes with exportin-t binding and also causes slow export (Table 3 and Fig. 6). Exportin-t binds all cytoplasmic tRNA species we and others have tested so far and thus apparently recognizes features common to all tRNAs (Arts et al., 1998a, 1998b; Kutay et al., 1998). Furthermore, exportin-t not only binds tRNA from mammals, but also from yeast, plants, and bacteria (Figs. 2 and 4), suggesting that the export receptor has adapted to ancient features of tRNA that already existed before evolution of nuclei and the nuclear transport machinery. Exportin-t shuttles between nucleus and cytoplasm (Arts et al., 1998a; Kutay et al., 1998) and for productive export cycles, it has to bind the tRNA in the nucleus and release it in the cytoplasm. As for all exportins known so far (Fornerod et al., 1997; Kutay et al., 1997, 1998; Stade et al., 1997; Arts et al., 1998a), this compartment specificity of binding is conferred by the cooperation of the exportin with the RanGTPase system.

Los1p (for loss of suppression) is the $S$. cerevisiae homolog of exportin-t (19\% sequence identity) and was identified nearly 20 years ago as a mutant that is de- ficient in the biogenesis of a suppressor tRNA (Hopper et al., 1980) and a role in tRNA export has been suspected since then. However, only recent advances in detection of tRNA trafficking in yeast (Bertrand et al., 1998) have made it possible to actually demonstrate that a los 1 deletion strain indeed accumulates IRNA in the nucleus (Sarkar \& Hopper, 1998). Furthermore, the tRNA-binding properties of Los1p (Hellmuth et al., 1998) resemble those of exportin-t; both interact with nuclear pore complexes (Simos et al., 1996; Hellmuth et al., 1998; Kutay et al., 1998) and are regulated by the RanGTPase system, suggesting that the two factors are indeed functional homologs. Nevertheless, Los 1 is not essential for viability (Hurt et al., 1987), indicating that tRNA can leave the nucleus also independently of Los1p. Although this might be accomplished by an alternative carrier, we would favor the explanation that tRNA is smaller than the diffusion limit of NPCs and it might simply diffuse out of the nucleus fast enough to allow survival. Because of the small dimensions of a yeast cell compared to a Xenopus oocyte, this simple diffusion should be more significant in yeast than in the Xenopus system.

Precise maps of the tRNA/exportin-t interaction have to await crystallographic analysis; however our data

TABLE 3.

\begin{tabular}{lc}
\hline tRNA ${ }^{\text {Lys3 }}$ point mutants & $\begin{array}{c}\text { Relative affinities } \\
\text { for exportin-t/RanGTP }\end{array}$ \\
\hline wild-type & 100 \\
A50C & 2.6 \\
A57U & 1.8 \\
A50C/G52C & 0.5 \\
C56U & 2.6 \\
G52C & 0.8
\end{tabular}

Relative affinities of the indicated tRNA species were determined as described in Table 1 and Figures $2 \mathrm{~B}$ and 4 . tRNAs were generated by in vitro transcription and had mature $5^{\prime}$ and $3^{\prime}$ ends. 


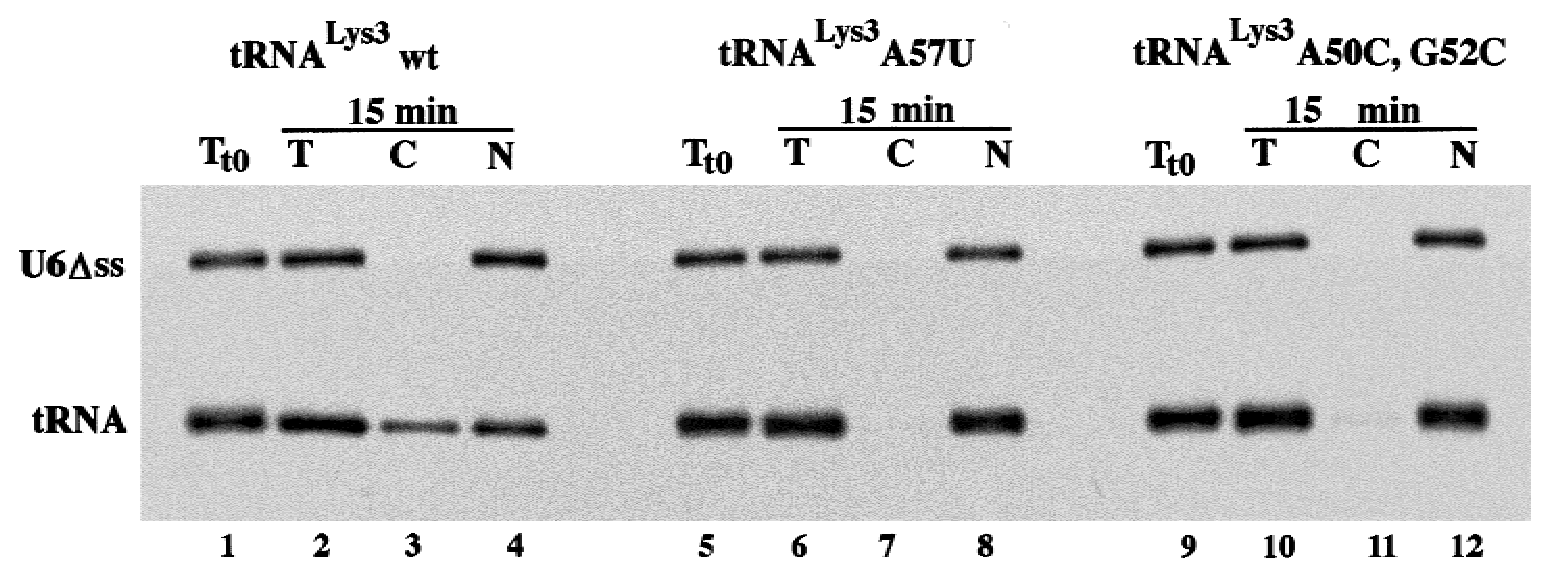

FIGURE 6. tRNA point mutants with low affinity for exportin-t are hardly exported from the nucleus. Wild-type or indicated point mutants of tRNA ${ }^{\text {Lys3 }}$ were injected into nuclei of Xenopus oocytes and their export was analyzed 15 min later as described in Figures 1 and 5. Note that wild-type tRNA ${ }^{\text {Lys3 } 3}$ was efficiently exported. The A57U and the A50C,G52C mutations not only abolished exportin-t binding of the tRNAs, but also severely affected their nuclear export.

already indicate that exportin-t might contact the terminal ends and the TUC loop of the tRNA. The anticodon loop and stem are probably not involved, as point mutations in the anticodon stem-loop have only a minor effect on tRNA export (Tobian et al., 1985). In contrast, mutations that disturb the secondary structure of the T stem or the tertiary tRNA structure essentially abolish exportin-t binding and hence export (Table 3 and Fig. 6), suggesting that the exportin-t must see the correct tRNA shape. We have analyzed the tRNA features required for high affinity interaction with exportin-t for the examples of tRNA ${ }^{\text {Tyr }}$ and tRNA ${ }^{\text {Lys } 3}$. A very recently published study arrived at similar conclusions for tRNA ${ }^{\text {Phe }}$ (Arts et al., 1998b). These authors further concluded from a combination of footprinting and binding interference that exportin-t recognizes and binds to the acceptor and the T $\Psi C$ arm of the mature tRNA.

A key demand on the nuclear export machinery is the proper coordination of tRNA maturation with export to avoid cytoplasmic accumulation of immature, nonfunctional tRNA species. We show here that exportin-t has a clear preference for mature tRNA (see Table 1). In particular, correctly processed $5^{\prime}$ and $3^{\prime}$ ends, including the $3^{\prime}$ CCA addition, are necessary for highaffinity interaction with exportin-t. Furthermore, modified nucleosides apparently play a role, although it is still unclear which particular modifications are important and whether they are directly recognized by the exportin or whether they simply maintain the correct tertiary tRNA structure.

Aminoacylation of tRNA inside the nucleus has recently been suggested to be a mechanism for functional proofreading of newly synthesized tRNAs (Lund \& Dahlberg, 1998). Although, this might contribute to the quality control prior to nuclear exit, it appears that the major proofreading event is binding of the tRNA to exportin-t, which can directly monitor correct tRNA shape, proper 5' and 3' ends, and base modifications. It remains to be seen to what extent aminoacylation of the tRNA influences binding to exportin-t, but it is clearly not a prerequisite. We and others have seen efficient exportin-t binding with nonacylated tRNAs (Arts et al., 1998a, 1998b; Kutay et al. 1998; this study). Consistent with that, Arts et al. (1998b) have shown that tRNA $^{\text {Phe }}$ mutants that are poorly aminoacylated are efficiently exported. Likewise, one of the tRNA ${ }^{T y r}$ species we are using (Fig. 5, lanes 9-12) also carries a double mutation (C1G, G72C) introduced to allow efficient in vitro transcription without the $5^{\prime}$ leader (Weber et al., 1996). This mutation has been reported to abolish aminoacylation by the eukaryotic tyrosyl-tRNA synthetase (Lee \& RajBhandary, 1991; Quinn et al., 1995). Nevertheless, the mature form of this mutant tRNA (see Fig. 5, lanes 10-12) was exported nearly as efficiently as a mature wild-type form (Fig. 5, e.g. lanes 5-8). Thus, aminoacylation might facilitate tRNA export, but is not obligatory for this process.

A surprising finding of this study is that exportin-t can perfectly bind intron-containing tRNA, provided the tRNA has correct $5^{\prime}$ and $3^{\prime}$ ends (Fig. 4). Consistent with that, unspliced tRNAs are efficiently exported when the rate of splicing is reduced (Fig. 5; see also Melton et al., 1980; Lund \& Dahlberg, 1998). The presence of an intron apparently does not significantly alter the structure of the mature part of the tRNA (Swerdlow \& Guthrie, 1984; Lee \& Knapp, 1985) and because the intron is inserted into the anticodon loop we would conclude that this part of the tRNA is not contacted by exportin-t. If exportin-t can bind and export intron-containing tRNA, why then are unspliced tRNAs not normally exported? Possible answers to this question obviously depend on the order of maturation steps of the tRNA. The prevailing view is that $5^{\prime}$ and $3^{\prime}$ end processing, most nucleoside modifications, and attachment of the $3^{\prime}$ CCA end usually oc- 
cur in vivo before splicing (see, e.g., Nishikura \& De Robertis, 1981; Stange \& Beier, 1987). This would imply that the tRNA is already in the high affinity form for exportin-t binding before splicing is completed. The coordination of splicing with export could be explained by a kinetic control, such as a substrate channeling from one processing enzyme to the next. Channeling would not only make processing more efficient, but also restrict the access of exportin-t to processing intermediates. A prediction from such a model is that the efficiency of one maturation step depends on the completion of the preceding one. Indeed we observed that splicing is inefficient when the tRNA was injected with an already correctly processed $5^{\prime}$ end (see Fig. 5).

A different answer would arise from the recent suggestion that tRNA splicing precedes $5^{\prime}$ end processing in Xenopus oocytes (Lund \& Dahlberg, 1998). This also would explain why a tRNA with a $5^{\prime}$ leader is spliced more efficiently than a tRNA that lacks this extension. In addition, in this case, splicing would have occurred before the tRNA has acquired correct $5^{\prime}$ and $3^{\prime}$ ends, that is, before export by exportin-t can occur. It is highly possible that the order of tRNA maturation events differs between different organisms and tRNA species and so might the coordination of tRNA maturation and export.

In Xenopus oocytes, exportin-t is rate-limiting for export of the mature tRNA, but not for splicing of, for example, tRNA $A^{\text {Tyr }}$ (E. Izaurralde \& G. Lipowsky, unpubl. observation). This situation appears quite different in yeast, where mutations in the exportin-t homolog Los1p cause a clear splicing defect (Hopper et al., 1980). This could be explained if intron-containing yeast tRNAs normally bind to Los $1 p$ prior to splicing and Los $1 p$ facilitates splicing by "presenting" the unspliced tRNA to the splicing machinery.

\section{MATERIALS AND METHODS}

\section{Antibodies}

The antibodies used for microinjection were raised in rabbits against the C-terminus of human exportin-t (Kutay et al., 1998) or against the $\mathrm{N}$-terminus of yeast CBC80 (control antibody). Both were affinity purified on the appropriate peptide columns, concentrated by ammonium sulfate precipitation, dialyzed against $2 \times$ PBS and adjusted to identical protein concentrations ( $\sim 20 \mathrm{mg} / \mathrm{mL})$. The anti-exportin-t antibody recognized a single band on total Xenopus oocyte extracts that had the expected size for exportin-t.

\section{Oocyte injection}

Oocyte injection and analysis of microinjected RNA by denaturing gel electrophoresis and autoradiography were essentially performed as described by Jarmolowski et al. (1994).

\section{Recombinant protein expression}

Exportin-t was expressed either with an N-terminal His-tag from pQE30 or with an N-terminal zz-tag (lgG binding domains from Protein A) at $23^{\circ} \mathrm{C}$, essentially as described by Kutay et al. (1998). Purification of N-His exportin-t was on nickel NTA agarose followed by chromatography on MonoQ. The expression and purification of the mutant Ran proteins (RanQ69L and RanT24N) have been described previously (Izaurralde et al., 1997).

\section{In vitro transcription}

All in vitro transcriptions were performed with T7 RNA polymerase. Preparation of ${ }^{32} \mathrm{P}$-labeled RNA probes for microinjections (Figs. 1, 5, and 6) was as described (Jarmolowski et al., 1994; Izaurralde et al., 1997). Large-scale preparation of unlabeled RNA was performed with the Ambion T7 Megashortscript kit. Templates were either plasmid DNA linearized with Fokl or BstNI (see Table 4) or gel-purified PCR products (see Table 5).

\section{Purification of native tRNA ${ }^{\text {Lys } 3}$}

Mature tRNA ${ }^{\text {Lys3 }}$ was purified from bovine liver according to Raba et al. (1979). Bovine and rabbit tRNA ${ }^{\text {Lys3 }}$ are identical in sequence.

\section{Binding assays}

Binding of zz-tagged exportin-t to IgG Sepharose has been described before (Kutay et al., 1998). Binding of tRNAs (500 pmol) from different organisms (calf, S. cerevisiae, E. coli, purchased from Boehringer Mannheim) to $10 \mu \mathrm{g}$ immobilized exportin-t was carried out in a total volume of $50 \mu \mathrm{L}$. One unit per milliliter RNasin, $0.1 \mathrm{mg} / \mathrm{mL}$ BSA, 500 pmol U6 $\Delta$ ssRNA (as an internal control for nonspecific binding) and $6 \mu \mathrm{M}$ of either RanQ69LGTP or RanT24NGDP were also present. After $3 \mathrm{~h}$ of incubation on ice, the samples were washed three times with $1 \mathrm{~mL}$ binding buffer $(50 \mathrm{mM} \mathrm{HEPES}-\mathrm{KOH}$, $200 \mathrm{mM} \mathrm{NaCl}, 5 \mathrm{mM} \mathrm{MgCl}_{2}$ ). Bound RNAs were eluted by addition of $200 \mu \mathrm{L}$ buffer (50 mM HEPES-KOH, $500 \mathrm{mM}$

TABLE 4. tRNA species obtained by in vitro transcription from linearized plasmids.

\begin{tabular}{|c|c|}
\hline $\begin{array}{l}\text { tRNA } \\
\text { (used in) }\end{array}$ & $\begin{array}{l}\text { Plasmid / linearized with } \\
\text { (Reference) }\end{array}$ \\
\hline Lys3 (wt) & pHtK10 / Fokl \\
\hline (Fig. 6; Tables 1, 2, 3) & (Schäfer et al., 1997) \\
\hline Tyr $+5^{\prime}$ leader + intron & pNtY1-T7 / BstNI \\
\hline (Fig. 5) & (Zerfass \& Beier, 1992) \\
\hline Tyr $+5^{\prime}$ leader & pNTY1 $1-\mathrm{T} 7$ / BstNI \\
\hline (Fig. 5) & (Zerfass \& Beier, 1992) \\
\hline Tyr + intron & pAtY3II*-T7 / BstNI \\
\hline (Fig. 5) & (Akama et al., 1997; Weber et al., 1996) \\
\hline Tyr A38C + intron & pAtY3II*-C38-T7 / BstNI \\
\hline (Fig. 5) & (Weber et al., 1996; Akama et al., 1997) \\
\hline
\end{tabular}


TABLE 5. tRNA species obtained by in vitro transcription from PCR products.

\begin{tabular}{|c|c|c|}
\hline $\begin{array}{l}\text { tRNA } \\
\text { (used in) }\end{array}$ & $\begin{array}{l}\text { PCR template } \\
\text { (Reference) }\end{array}$ & Primers used for PCR \\
\hline Lys3 mature ends & $\mathrm{pHtK9}$ & wt-T7ss: 5'-TAATACGACTCACTATAGCCCGGATA-3' \\
\hline (Table 1) & (Schäfer et al., 1997) & wt-as: 5'-TGGCGCCCGAACAGGGAC-3' \\
\hline Lys $3+5^{\prime}$ extension & $\mathrm{pHtK9}$ & T7ss:5'-TAATACGACTCACTATAGTAACCGTTGGCCCG-3' \\
\hline (Table 1) & (Schäfer et al., 1997) & wt-as: as above \\
\hline Lys3 $+3^{\prime}$ extension & $\mathrm{pHtK9}$ & wt-T7ss: as above \\
\hline (Table 1) & (Schäfer et al., 1997) & 3' + 10as: 5'-GTTGGCCCTTTGGCGCCCG-3' \\
\hline Lys3 without CCA & $\mathrm{pHtK9}$ & wt-T7ss: as above \\
\hline (Table 1) & (Schäfer et al., 1997) & $\triangle$ CCA-as: 5'-CGCCCGAACAGGGACTTGAA-3' \\
\hline Lys3-wt & $\mathrm{pHtK} 10$ & wt-T7ss: as above \\
\hline (Table 2) & (Schäfer et al., 1997) & wt-as: as above \\
\hline Lys3-wt: CC & $\mathrm{pHtK} 10$ & wt-T7ss: as above \\
\hline (Table 2) & (Schäfer et al., 1997) & CC-as: 5'-GGCGCCCGAACAGGGACTT-3' \\
\hline Lys3-wt: C & $\mathrm{pHtK} 10$ & wt-T7ss: as above \\
\hline (Table 2) & (Schäfer et al., 1997) & C-as: 5'-GCGCCCGAACAGGGACTTG-3' \\
\hline Lys3-wt: UUU & $\mathrm{pHtK} 10$ & wt-T7ss: as above \\
\hline (Table 2) & (Schäfer et al., 1997) & UUU-as: 5'-AAACGCCCGAACAGGGAC-3' \\
\hline Lys3 A50C & $\mathrm{pHtK} 19$ & wt-T7ss: as above \\
\hline (Table 3) & (Schäfer et al., 1997) & wt-as: as above \\
\hline Lys3 G52C & $\mathrm{pHtK} 36$ & wt-T7ss: as above \\
\hline (Table 3) & (Schäfer et al., 1997) & wt-as: as above \\
\hline Lys3 A50C/G52C & $\mathrm{pHtK} 15$ & wt-T7ss: as above \\
\hline (Fig. 6; Table 3) & (Schäfer et al., 1997) & wt-as: as above \\
\hline Lys3 C56U & $\mathrm{pHtK} 44$ & wt-T7ss: as above \\
\hline (Table 3) & (Schäfer et al., 1997) & wt-as: as above \\
\hline Lys3 A57U & $\mathrm{pHtK} 46$ & wt-T7ss: as above \\
\hline (Fig. 6; Table 3) & (Schäfer et al., 1997) & wt-as: as above \\
\hline Tyr-wt (intron) & pNtY1-T7 & Tyr-T7ss: 5'-TAATACGACTCACTATAGCGACCTTAGCTC-3' \\
\hline (Fig. 4) & (Zerfass \& Beier, 1992) & Tyr-as: 5'-TGGTGCGACCTACCGGATTCGAAC-3' \\
\hline Tyr-wt & $\mathrm{pNtY} 1 \Delta-\mathrm{T} 7$ & Tyr-T7ss: as above \\
\hline (Fig. 4) & (Zerfass \& Beier, 1992) & Tyr-as: as above \\
\hline
\end{tabular}

$\mathrm{NaCl}, 5 \mathrm{mM} \mathrm{MgCl} 2$ ) and $200 \mu \mathrm{L}$ phenol/chloroform. RNAs were precipitated from the aqueous phase with ethanol. One tenth of the bound fractions were analyzed by denaturing gel electrophoresis and compared with 1/20 of the starting material after SYBR-GOLD (Molecular Probes) staining.

Kinetic RanGTPase assays were performed as described (Bischoff et al., 1995; Kutay et al., 1998). Note that all tRNA species used for the binding experiments in this study were nonacylated.

\section{ACKNOWLEDGMENTS}

We would like to thank Dr. H. Fried for critical reading of the manuscript, Petra Schwarzmaier for excellent technical help and the Deutsche Forschungsgemeinschaft (SFB 352) and the Human Frontier Science Programme Organization (grant RG0198/1998M) for financial support.

Received November 30, 1998; returned for revision January 6, 1999; revised manuscript received January 14, 1999

\section{REFERENCES}

Abelson J, Trotta CR, Li H. 1998. tRNA splicing. J Biol Chem 273:12685-12688.
Akama K, Nass A, Junker V, Beier H. 1997. Characterization of nuclear tRNA(Tyr) introns: Their evolution from red algae to higher plants. FEBS Lett 417:213-218.

Arts GJ, Fornerod M, Mattaj IW. 1998a. Identification of a nuclear export receptor for tRNA. Curr Biol 8:305-314.

Arts GJ, Kuersten S, Romby P, Ehresmann B, Mattaj IW. 1998b. The role of exportin-t in selective nuclear export of mature tRNA. EMBO J 17:7430-7441.

Belfort M, Weiner A. 1997. Another bridge between kingdoms: tRNA splicing in archaea and eukaryotes. Cell 89:1003-1006.

Bertrand E, Houser-Scott F, Kendall A, Singer RH, Engelke DR. 1998. Nucleolar localization of early tRNA processing. Genes \& Dev 12:2463-2468.

Bischoff FR, Krebber H, Smirnova E, Dong WH, Ponstingl H. 1995. Coactivation of RanGTPase and inhibition of GTP dissociation by Ran GTP binding protein RanBP1. EMBO J 14:705-715.

Björk GR. 1995. Biosynthesis and function of modified nucleosides. In: Söll D, RajBhandary UL, eds. tRNA: Structure, biosynthesis, and function. Washington, DC: ASM Press. pp 165-206.

Boelens WC, Palacios I, Mattaj IW. 1995. Nuclear retention of RNA as a mechanism for localization. RNA 1:273-283.

Dahlberg JE, Lund E. 1998. Functions of the GTPase Ran in RNA export from the nucleus. Curr Opin Cell Biol 10:400-408.

De Robertis EM, Black P, Nishikura K. 1981. Intranuclear location of the tRNA splicing enzymes. Cell 23:89-93.

Deutscher MP. 1995. tRNA processing nucleases. In: Söll D, RajBhandary UL, eds. tRNA: Structure, biosynthesis, and function. Washington, DC: ASM Press. pp 51-66.

Dirheimer G, Keith G, Dumas P, Westhof E. 1995. Primary, secondary, and tertiary structures of tRNA. In: Söll D, RajBhandary UL, eds. tRNA: Structure, biosynthesis, and function. Washington, DC: ASM Press. pp 93-126. 
Floer M, Blobel G. 1996. The nuclear transport factor karyopherin $\beta$ binds stoichiometrically to Ran-GTP and inhibits the Ran GTPase activating protein. J Biol Chem 271:5313-5316.

Fornerod M, Ohno M, Yoshida M, Mattaj IW. 1997. Crm1 is an export receptor for leucine-rich nuclear export signals. Cell 90:10511060.

Görlich D, Panté N, Kutay U, Aebi U, Bischoff FR. 1996. Identification of different roles for RanGDP and RanGTP in nuclear protein import. EMBO J 15:5584-5594.

Hellmuth K, Lau DM, Bischoff FR, Kunzler M, Hurt E, Simos G. 1998. Yeast los $1 p$ has properties of an exportin-like nucleocytoplasmic transport factor for tRNA. Mol Cell Biol 18:6374-6386.

Hopper AK, Schultz LD, Shapiro RA. 1980. Processing of intervening sequences: A new yeast mutant which fails to excise intervening sequences from precursor tRNAs. Cell 19:741-751.

Hurt DJ, Wang SS, Lin YH, Hopper AK. 1987. Cloning and characterization of LOS1, a Saccharomyces cerevisiae gene that affects tRNA splicing. Mol Cell Biol 7:1208-1216.

Izaurralde E, Adam SA. 1998. Transport of macromolecules between the nucleus and the cytoplasm. RNA 4:351-364.

Izaurralde E, Kutay U, von Kobbe C, Mattaj IW, Görlich D. 1997. The asymmetric distribution of the constituents of the Ran system is essential for transport into and out of the nucleus. EMBO $J$ 16:6535-6547.

Jarmolowski A, Boelens WC, Izaurralde E, Mattaj IW. 1994. Nuclear export of different classes of RNA is mediated by specific factors. J Cell Biol 124:627-635.

Kutay U, Bischoff FR, Kostka S, Kraft R, Görlich D. 1997. Export of importin alpha from the nucleus is mediated by a specific nuclear transport factor. Cell 90:1061-1071.

Kutay U, Lipowsky G, Izaurralde E, Bischoff FR, Schwarzmaier P, Hartmann E, Görlich D. 1998. Identification of a tRNA-specific nuclear export receptor. Mol Cell 1:359-369.

Lee CP, RajBhandary UL. 1991. Mutants of Escherichia coli initiator tRNA that suppress amber codons in Saccharomyces cerevisiae that are aminoacylated with tyrosine by yeast extracts. Proc Natl Acad Sci USA 88:11378-11382.

Lee MC, Knapp G. 1985. Transfer RNA splicing in Saccharomyces cerevisiae. Secondary and tertiary structures of the substrates. $J$ Biol Chem 260:3108-3115.

Lund E, Dahlberg JE. 1998. Proofreading and aminoacylation of tRNAs before export from the nucleus. Science 282:2082-2085.

Mattaj IW, Englmeier L. 1998. Nucleocytoplasmic transport: The soluble phase. Ann Rev Biochem 67:265-306.

Melton DA, De Robertis EM, Cortese R. 1980. Order and intracellular location of the events involved in the maturation of a spliced tRNA. Nature 284:143-148.

Nishikura K, De Robertis EM. 1981. RNA processing in microinjected Xenopus oocytes. Sequential addition of base modifications in the spliced transfer RNA. J Mol Biol 145:405-420.

Pederson T. 1998. The plurifunctional nucleolus. Nucleic Acids Res 26:3871-3876
Quinn CL, Tao N, Schimmel P. 1995. Species-specific microhelix aminoacylation by a eukaryotic pathogen tRNA synthetase dependent on a single base pair. Biochemistry 34:12489-12495.

Raba M, Limburg K, Burghagen M, Katze JR, Simsek M, Heckman JE, Rajbhandary UL, Gross HJ. 1979. Nucleotide sequence of three isoaccepting lysine tRNAs from rabbit liver and SV40transformed mouse fibroblasts. Eur J Biochem 97:305-318.

Sarkar S, Hopper AK. 1998. tRNA nuclear export in Saccharomyces cerevisiae: In situ hybridization analysis. Mol Biol Cell 9:30413055.

Schäfer S, Heumann H, Gross HJ. 1997. Mammalian tRNA(Lys)3 and pre-tRNA(Lys) 3 variants as primers and inhibitors of viral cDNA synthesis by HIV reverse transcriptase in vitro. Nucleic Acids Symp Ser 291-292.

Simos G, Tekotte H, Grosjean H, Segref A, Sharma K, Tollervey D, Hurt EC. 1996. Nuclear pore proteins are involved in the biogenesis of functional tRNA. EMBO J 15:2270-2284.

Sprague KU. 1995. Transcription of eukaryotic tRNA genes. In: Söll D, RajBhandary UL, eds. tRNA: Structure, biosynthesis, and function. Washington, DC: ASM Press. pp 31-50.

Sprinzl M, Hartmann T, Weber J, Blank J, Zeidler R. 1989. Compilation of tRNA sequences and sequences of tRNA genes. Nucleic Acids Res 17 (Suppl):1-172.

Stade K, Ford CS, Guthrie C, Weis K. 1997. Exportin 1 (Crm1p) is an essential nuclear export factor. Cell 90:1041-1050.

Stange N, Beier H. 1987. A cell-free plant extract for accurate pre-tRNA processing, splicing and modification. EMBO J 6:2811-2818.

Swerdlow H, Guthrie C. 1984. Structure of intron-containing tRNA precursors: Analysis of solution conformation using chemical and enzymatic probes. J Biol Chem 259:5197-5207.

Tobian JA, Drinkard L, Zasloff M. 1985. tRNA nuclear transport: Defining the critical regions of human $\operatorname{tRNA}_{i}^{\text {Met }}$ by point mutagenesis. Cell 43:415-422.

Weber U, Beier H, Gross HJ. 1996. Another heritage from the RNA world: Self-excision of intron sequence from nuclear pre-tRNAs. Nucleic Acids Res 24:2212-2219.

Westaway SK, Abelson J. 1995. Splicing of tRNA precursors. In: Söll D, RajBhandary UL, eds. tRNA: Structure, biosynthesis, and function. Washington, DC: ASM Press. pp 79-92.

Zasloff M. 1983. tRNA transport from the nucleus in a eukaryotic cell: Carrier-mediated translocation process. Proc Natl Acad Sci USA 80:6436-6440.

Zasloff M, Rosenberg M, Santos T. 1982a. Impaired nuclear transport of a human variant tRNA $A_{i}^{\text {Met }}$. Nature 300:81-84.

Zasloff M, Santos T, Hamer DH. 1982b. tRNA precursor transcribed from a mutant human gene inserted into a SV40 vector is processed incorrectly. Nature 295:533-535.

Zerfass K, Beier H. 1992. Pseudouridine in the anticodon GUA of plant cytoplasmic tRNA(Tyr) is required for UAG and UAA suppression in the TMV-specific context. Nucleic Acids Res 20:59115918. 